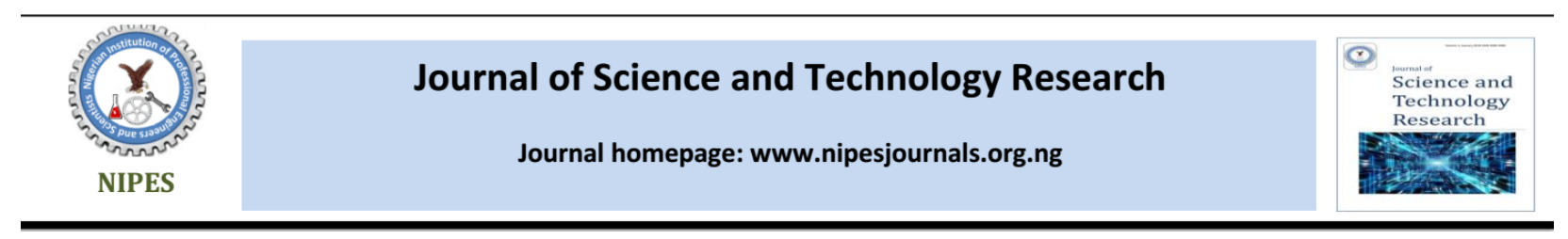

\title{
Solving Predator-Prey Model Using Maple 18 Coded Variational Iteration Method (VIM)
}

\section{Falade, K. I. ${ }^{a^{*}}$, Salisu, U. ${ }^{b}$, Ayodele, V.I ${ }^{c}$}

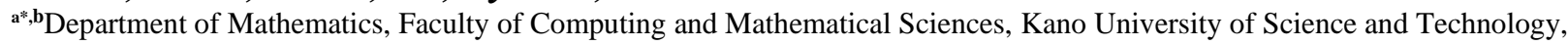
P.M.B 3244 Wudil Kano State, Nigeria.

'Department of Computer Science and Mathematics, Faculty of Science, Nigeria Police Academy, Wudil Kano State, Nigeria.

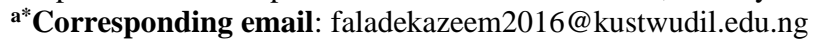

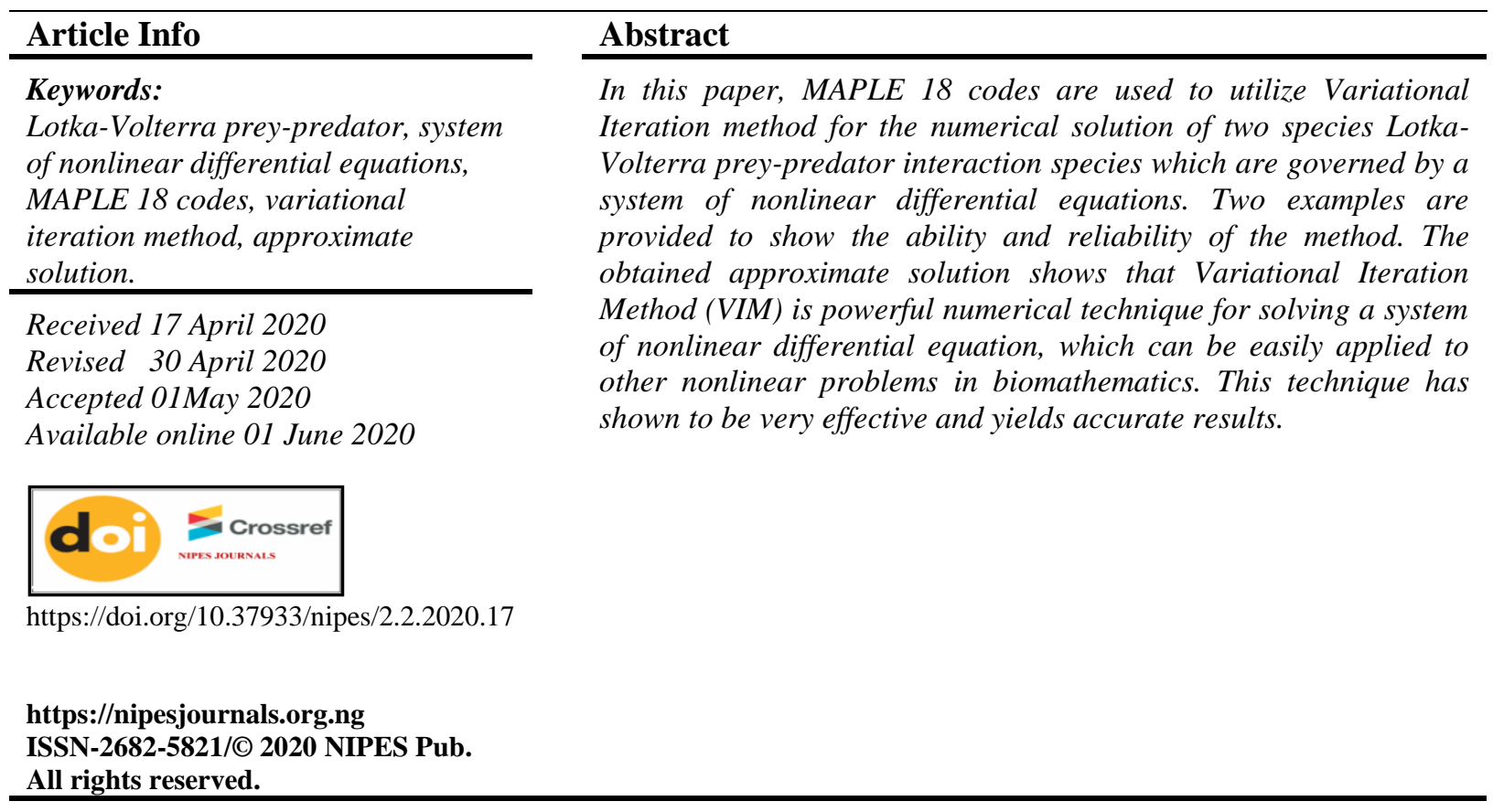

\section{Introduction}

System of differential equation has a wide field in pure and applied mathematics such as transportation problem, economics mathematics, meteorology, biomathematics and engineering. All of these disciplines are concerned with the properties of differential equations of various types which emphasizes the rigorous justification and interpretation of natural phenomena. It plays an important role in modelling virtually every physical, technical, or biological process, from celestial motion, to bridge design, to interactions between neurons. Nonlinear differential equations are used for describing many phenomena in the real world as prey predator interactions. Prey predator models are classified as one of the most important applications in applied mathematics, thus many numerical and semi-analytical methods are developed for finding the solution of these problems by many researchers $[1,2,3]$.

Relevant biological models may involve interactions (from the biochemical to the ecosystem level). One of the first interactions model in population dynamics was introduced in the beginning of the 20th century by Alfred Lotka, an American biophysicist (1925) and Vito Volterra, an Italian mathematician (1926) models interactions between preys and predators. The population sizes are denoted by $x(t)$ preys and $y(t)$ predators at time $t \geq 0$. He assumed that population change of one 
specie depends on its current population, reproduction rate and interactions with other species (predator and prey) [4].

In recent years, research on Lotka-Volttera system of nonlinear differential equations have been extensively investigated in the literature. [5] proposed a new method for the explicit integration of Lotka-Volterra equations, the authors in [6] solved stochastic Lotka-Volterra equations via operational matrices, a study on competitive Lotka-Volterra model in random environments was examined and studied by [7]. [8] studied and observed the population dynamics of a three-species Lotka-Volterra model of two predators and their prey, [9] applied differential transformation method for solving prey predator model with holling type I. [10] studied a series solutions of two species Lotka-Volterra Equations by Adomian Decomposition and Homotopy Perturbation Methods and [11] studied Predator-Prey system in a polluted environment.

\subsection{Lotka-Voltarra Model}

Lotka-Volterra model also known as the predator-prey equations, in deterministic subclasses, are well-known and have been an active area of research concerning ecological population modeling and economic modeling. These type of equations is so attractive in the terms of population dynamics of species competing (conflict) and the logistic population model. Thus, the Lotka-Volterra model in case of two species is a prey predator equation which is defined as follows:

$$
\left\{\begin{array}{l}
\frac{d x}{d t}=\alpha x(t)-\beta x(t) y(t) \\
\frac{d y}{d t}=-\omega y(t)+\tau x(t) y(t)
\end{array}\right.
$$

subject to initial conditions

$$
\left\{\begin{array}{l}
x\left(t_{0}\right)=A \\
y\left(t_{0}\right)=B
\end{array}\right.
$$

where the function $x(t)$ represents the populations of prey at time $t$, and also the function $y(t)$ represents the populations of predator at time $t$. All of the parameters $\alpha, \beta, \omega, \tau$ are non-negative constants. The parameter $\alpha$ represents the per capita reduction in prey per predator. The parameter $\beta$ represents death rate per encounter of prey due to predation. Moreover if $\beta$ is the only decreasing factor for the prey population, then prey will be eaten by predators. The parameter $\omega$ represents the per capita increase in predator per prey, moreover if $\omega$ is the only increasing factor for the predator population, then the population growth is proportional to the food available. The parameter $\tau$ represents mortality rate of predator and $A, B$ are constants.

In reality, if the prey population is large, the predators will have more food to support a larger population. However, when the predator population grows too large, the prey begins to die off. This will result in a decrease in the predators. This trend continues as time goes on, implying a stable coexistence of the two populations.

The main objectives of this paper is to present and employ a MAPLE 18 software codes for variational iteration method proposed in [12] and to overcome the mathematical stress of integral involve in implementation of VIM.

This paper is organized as follows. In section 1, we introduced briefly the Lotka-Volterra model and their parameters notation related to Predator-prey interaction behaviors. Moreover, stability and equilibrium of the model are discussed. In section 2, we discussed and formulate MAPLE 18 Variation iteration scheme. Section 3, the variational iterative method was applied to solve predatorprey Equation (1) while in section 4, the numerical results table and graphs are reported. Finally, section 5 provides the discussion and conclusion. 


\subsection{The Dynamic Behavior of the Lotka-Volterra Model}

One of the main properties of dynamic systems is stability. The stability is studied to determine some properties of solutions or system of differential equations. Consequently the dynamic behavior of the model will be discussed. For the model equilibrium points, we set the right hand side of (1) to zero.

$$
\begin{aligned}
& (\alpha-\beta y(t)) x(t)=0 \\
& (-\omega+\tau x(t)) y(t)=0
\end{aligned}
$$

to obtain $x=0, y=0$ or $x=\frac{\omega}{\tau}, y=\frac{\alpha}{\beta}$. Then system (1) has

i. the trivial equilibrium, $E_{0}\left(x_{0}, y_{0}\right)=(0,0)$,

ii. the non-trivial equilibrium point, $E^{*}\left(x^{*}, y^{*}\right)=\left(\frac{\omega}{\tau}, \frac{\alpha}{\beta}\right)$.

To investigate the stability of each equilibrium point, we evaluate the Jacobian matrix of (1) at each of these equilibrium. The Jacobian matrix of system (1) is given by

$$
J(x, y)=\left(\begin{array}{cc}
\alpha-\beta y & -\beta x \\
\tau y & -\omega+\tau x
\end{array}\right)
$$

\subsection{At the Trivial Equilibrium}

We obtain from (4) that

$$
J\left(E_{0}\right)=\left(\begin{array}{cc}
\alpha & 0 \\
0 & -\omega
\end{array}\right) .
$$

Thus the two eigenvalues of $J\left(E_{0}\right)$ are $\lambda_{1}=\alpha$ and $\lambda_{2}=-\omega$. Therefore, $E_{0}$ is a saddle point since one of the eigenvalues is positive and the other is negative.

The corresponding eigenvectors are the two axes $x$ and $y$. Then using the standard computation of eigenvectors, we have for the eigenvector associated with $\lambda_{1}=\alpha$

$$
\left(\begin{array}{cc}
\alpha & 0 \\
0 & -\omega
\end{array}\right)\left(\begin{array}{l}
x \\
y
\end{array}\right)=\alpha\left(\begin{array}{l}
x \\
y
\end{array}\right)
$$

so that $(\alpha+\omega) y=0$ and so, $y=0$ which is the $x$-axis. Therefore, the $x$ component of a perturbation away from but still near the $E_{0}$ will grow exponentially at a rate $\alpha$.

Similarly, $x=0$, which is the $y-$ axis, is the eigenvector associated with $\lambda_{2}=-\omega$. Therefore, the $y$ component of a perturbation away from but still near the $E_{0}$ will shrink exponentially at a rate $-\omega$.

\subsection{At the non-trivial equilibrium (Coexistence Equilibrium)}

The Jacobian matrix in (4) becomes:

$$
J\left(E^{*}\right)=\left(\begin{array}{cc}
0 & -\beta \frac{\omega}{\tau} \\
\tau \frac{\alpha}{\beta} & 0
\end{array}\right)
$$

The eigenvalues of $J\left(E^{*}\right)$ are $\lambda_{1,2}= \pm i \sqrt{\alpha \omega}$. This show that the trace of this matrix is zero and the determinant is $\alpha \omega>0$. Thus $E^{*}$ is a centre and is balanced at the knife-edge between stable and unstable oscillations. Therefore system (1) is structurally unstable since any slight change to the structure of the equations, especially changing the nonlinear terms, could tip the balance between stability or instability, depending on how changes to the structure of the equations affects the real part of the eigenvalues. In a structurally unstable system, slight modifications to the form of the equations alter the stability.

The counterclockwise circulation of vectors near the coexistence equilibrium $E^{*}$ is as a result of the purely imaginary eigenvalues of $\left(E^{*}\right)$. Any perturbation near this equilibrium causes the system to oscillate around it infinitely in a closed orbit, neither growing away from the equilibrium nor returning to it. The larger the perturbation, the greater the amplitude of the circulation, see Figures 4 and 6 for example. 


\section{Description of the Variation Iteration Method (VIM) and Solution Approach}

The basic idea of the variational iteration method is to construct an iteration procedures based on correction functional that include a generalized Lagrange multiplier [13-14]. The VIM was proposed where the value of the multiplier was chosen using variational theory so that each improves the accuracy of the solution [15].The initial approximation i.e. trial function usually includes unknown coefficient which can be determined to satisfy any boundary and initial conditions. VIM does not require specific transformation for nonlinear terms as required by other techniques and is now widely used by many researchers to study autonomous ordinary differential equation, Integro-differential systems, Linear Helmholtz partial differential equation and other fields [16-21]. In this method the solution is given in an infinite series usually convergent to an accurate solution. According to the variational iteration method we consider the following general differential equation of the form:

$$
L p+N p=g(t)
$$

where $\mathrm{L}$ is a linear operator $N$ is a nonlinear operator and $g(t)$ is an inhomogeneous term. We can construct a correctional function as follows

$$
x_{n+1}=x_{n}(t) \int_{0}^{t} \lambda\left\{L x_{n}(s)+N \overline{x_{n}}(s)-g(s)\right\} d s
$$

Where $\lambda$ is a Lagrangian multiplier which can be identified optimally via variational theory [22]. The subscript $n$ denotes the $n$th approximation Consider the stationary condition of the above correction functional then the Lagrange multiplier can be expressed as

$$
\lambda_{i}(w)=\frac{(-1)^{q}}{(q-1) !}(q-t)^{q-1}
$$

Where $\mathrm{q}$ is the highest order of the differential equation.

\subsection{Maple 18 Coded Variational Iteration Method}

In order to formulate the general variational iteration approach on MAPLE 18 software, we consider Equations (1) and (2) and develop the VIM schemes as follows:

\section{MAPLE 18 Coded Variational Iteration Scheme}




$$
\left\{\begin{array}{c}
\text { restart }: \\
\alpha:=R_{1} ; \beta:=R_{2} ; \omega:=R_{3} ; \tau:=R_{4} ; \lambda:=(-1) ; \\
x_{0}:=A ; y_{0}:=B ; a_{0}:=\operatorname{diff}\left(x_{0}, t\right) ; a_{1}:=\operatorname{diff}\left(y_{0}, t\right) ; \\
x_{1}:=x_{0}+(\lambda) * \operatorname{int}\left\{\left(a_{0}-\left(\alpha * x_{0}\right)+\left(\beta * x_{0} * y_{0}\right)\right), t=0 \ldots t\right\} ; \\
x_{11}:=\operatorname{value}\left(x_{1}\right) ; b_{0}:=\operatorname{diff}\left(x_{11}, t\right) ; \\
y_{1}:=y_{0}+(\lambda) * \operatorname{int}\left\{\left(a_{1}+\left(\omega * y_{0}\right)-\left(\tau * x_{0} * y_{0}\right)\right), t=0 \ldots t\right\} ; \\
y_{11}:=\operatorname{value}\left(y_{1}\right) ; b_{1}:=\operatorname{diff}\left(y_{11}, t\right) ; \\
x_{2}:=x_{11}+(\lambda) * \operatorname{int}\left\{\left(b_{0}-\left(\alpha * x_{11}\right)+\left(\beta * x_{11} * y_{11}\right)\right), t=0 \ldots t\right\} ; \\
x_{12}:=\operatorname{value}\left(x_{2}\right) ; c_{0}:=\operatorname{diff}\left(x_{12}, t\right) ; \\
y_{2}:=y_{11}+(\lambda) * \operatorname{int}\left\{\left(b_{1}+\left(\omega * y_{11}\right)-\left(\tau * x_{11} * y_{11}\right)\right), t=0 \ldots t\right\} ; \\
y_{12}:=\operatorname{value}\left(y_{2}\right) ; c_{1}:=\operatorname{diff}\left(y_{12}, t\right) ; \\
x_{3}:=x_{12}+(\lambda) * \operatorname{int}\left\{\left(c_{0}-\left(\alpha * x_{12}\right)+\left(\beta * x_{12} * y_{12}\right)\right), t=0 \ldots t\right\} ; \\
x_{13}:=\operatorname{value}\left(x_{3}\right) ; d_{0}:=\operatorname{diff}\left(x_{13}, t\right) ; \\
y_{3}:=y_{12}+(\lambda) * \operatorname{int}\left\{\left(c_{1}+\left(\omega * y_{12}\right)-\left(\tau * x_{11} * y_{12}\right)\right), t=0 \ldots t\right\} ; \\
y_{13}:=\operatorname{value}\left(y_{3}\right) ; d_{1}:=\operatorname{diff}\left(y_{13}, t\right) ; \\
\vdots \\
\vdots \\
\vdots \\
y_{m}:=y_{1 m-1}+(\lambda) * \operatorname{int}\left\{\left(s_{1}+\left(\omega * y_{1 m-1}\right)-\left(\tau * x_{1 m-1} * y_{1 m-1}\right)\right), t=0 \ldots t\right\} ; \\
y_{1 m}:=\operatorname{value}\left(y_{m}\right) ; z_{1}:=\operatorname{diff}\left(y_{1 m}, t\right) ; \\
x(t):=\operatorname{evalf}\left(x_{1 m}\right) ; \\
y(t):=\operatorname{evalf}\left(y_{1 m}\right) ; \\
x_{1 m-1}+(\lambda) * \operatorname{int}\left\{\left(s_{0}-\left(\alpha * x_{1 m-1}\right)+\left(\beta * x_{1 m-1} * y_{1 m-1}\right)\right), t=0 \ldots t\right\} ;
\end{array}\right.
$$

where $m$ is the number of iterations, $R_{1}, R_{2}, R_{3}, R_{4}$ are parameter constants and $A, B$ are constants. Thus, $x(t)$ and $y(t)$ are the series solutions at $m t h$ iteration.

\section{Numerical Experiment}

In this section, we examine the predator and prey growth/decay of the two species (self-interaction) as well as their interaction. We investigate bahaviours of the parameters $\alpha, \beta, \omega$ and $\tau$ which play a key role in determining the system behaviours. Two examples are considered subject to specific initial condition of the model. Using the MAPLE 18 coded Variation Iteration scheme, we obtained the numerical solution for Equation (1) and initial condition (2) at iteration step $m=10$ as shown in Tables 1 to 6 .

Example 1 Suppose $\alpha$ is the per capita reduction in prey per predator and $\beta$ is the death rate per encounter of prey due to predation [23].

$$
\left\{\begin{array} { l } 
{ \text { when } ( \alpha < \beta ) } \\
{ \alpha = 3 . 0 2 9 8 5 0 } \\
{ \beta = 4 . 0 9 4 1 3 2 } \\
{ \omega = 1 . 9 6 7 2 1 7 } \\
{ \tau = 2 . 2 9 5 9 4 2 } \\
{ \lambda = - 1 . 0 0 0 0 0 } \\
{ x _ { 0 } = 1 . 1 8 7 1 0 0 } \\
{ y _ { 0 } = 0 . 7 4 0 0 4 7 }
\end{array} \quad \left\{\begin{array} { l } 
{ \alpha = 4 . 0 9 4 1 3 2 } \\
{ \beta = 3 . 0 2 9 8 5 0 } \\
{ \tau = 1 . 9 6 7 2 1 7 } \\
{ x _ { 0 } = 1 . 1 8 7 1 0 0 } \\
{ y _ { 0 } = 0 . 7 4 0 0 4 7 }
\end{array} \quad \left\{\begin{array}{l}
\alpha=3.029850 \\
\beta=3.029850 \\
\omega=1.967217 \\
\tau=2.295942 \\
\lambda=-1.00000 \\
x_{0}=1.187100 \\
y_{0}=0.740047
\end{array}\right.\right.\right.
$$

Substitute the above parameters into algorithm (11), we have the following solutions: 
Table 1: $(\alpha<\beta)$

\begin{tabular}{|c|c|c|c|c|}
\hline $\mathrm{t}$ & $\begin{array}{c}\text { Analytical } \\
\text { Solution } x(t)\end{array}$ & $\begin{array}{c}\text { VIM } \\
\text { Solution } x(t)\end{array}$ & $\begin{array}{c}\text { Analytical } \\
\text { Solution } y(t)\end{array}$ & $\begin{array}{c}\text { VIM } \\
\text { Solution } y(t)\end{array}$ \\
\hline 0.0 & 1.187100000000 & 1.187100000000 & 0.7400470000000 & 0.7400470000000 \\
\hline 0.1 & 1.173293314174 & 1.173293331000 & 0.7975048380086 & 0.7975047610000 \\
\hline 0.2 & 1.132681174388 & 1.132681233000 & 0.8540331977419 & 0.8540330912000 \\
\hline 0.3 & 1.069749554336 & 1.069749539000 & 0.9036493434085 & 0.9036492527000 \\
\hline 0.4 & 0.992376686741 & 0.992376610300 & 0.9407098212139 & 0.9407097698000 \\
\hline 0.5 & 0.909715706923 & 0.909715551500 & 0.9612974253317 & 0.9612976897000 \\
\hline 0.6 & 0.829963573965 & 0.829961925000 & 0.9640576734517 & 0.9640598752000 \\
\hline 0.7 & 0.758989599935 & 0.758979098800 & 0.9501583685533 & 0.9501676352000 \\
\hline 0.8 & 0.700090581059 & 0.700045711900 & 0.9225636508131 & 0.9225907487000 \\
\hline 0.9 & 0.654510497041 & 0.654363278700 & 0.8850829288848 & 0.8851423567000 \\
\hline 1.0 & 0.622205729269 & 0.621805168400 & 0.8415741110666 & 0.8416743875000 \\
\hline
\end{tabular}

Table 2: $(\alpha>\beta)$

\begin{tabular}{|c|c|c|c|c|}
\hline $\mathrm{t}$ & $\begin{array}{c}\text { Analytical } \\
\text { Solution } x(t)\end{array}$ & $\begin{array}{c}\text { VIM } \\
\text { Solution } x(t)\end{array}$ & $\begin{array}{c}\text { Analytical } \\
\text { Solution } y(t)\end{array}$ & $\begin{array}{c}\text { VIM } \\
\text { Solution } y(t)\end{array}$ \\
\hline 0.0 & 1.187100000000 & 1.187100000000 & 0.7400470000000 & 0.740047000000 \\
\hline 0.1 & 1.413308431481 & 1.413308491000 & 0.8192192423347 & 0.819219212200 \\
\hline 0.2 & 1.629422801606 & 1.629422522000 & 0.9548299457312 & 0.954830107800 \\
\hline 0.3 & 1.783965142331 & 1.783964837000 & 1.1626432476204 & 1.162643502000 \\
\hline 0.4 & 1.812364112012 & 1.812363259000 & 1.4474459753223 & 1.447445942000 \\
\hline 0.5 & 1.674587231402 & 1.674575344000 & 2.0850176428230 & 2.085017642720 \\
\hline 0.6 & 1.401596690416 & 1.401562584000 & 2.0850176428292 & 2.085017641320 \\
\hline 0.7 & 1.085902388192 & 1.086180815000 & 2.2783956366840 & 2.278395618000 \\
\hline 0.8 & 0.811351279883 & 0.811351460690 & 2.3239540305871 & 2.323951416000 \\
\hline 0.9 & 0.610020458917 & 0.610020361200 & 2.2440744293156 & 2.244074124000 \\
\hline 1.0 & 0.476079350030 & 0.476079236100 & 2.0857367907384 & 2.085736623000 \\
\hline
\end{tabular}

Table 3: $(\alpha=\beta)$

\begin{tabular}{|c|c|c|c|c|}
\hline $\mathrm{t}$ & $\begin{array}{c}\text { Analytical } \\
\text { Solution } x(t)\end{array}$ & $\begin{array}{c}\text { VIM } \\
\text { Solution } x(t)\end{array}$ & $\begin{array}{c}\text { Analytical } \\
\text { Solution } y(t)\end{array}$ & $\begin{array}{c}\text { VIM } \\
\text { Solution } y(t)\end{array}$ \\
\hline 0.0 & 1.187100000000 & 1.187100000000 & 0.740047000000 & 0.740047000000 \\
\hline 0.1 & 1.272187665251 & 1.272187275000 & 0.806474171253 & 0.806474407600 \\
\hline 0.2 & 1.331974163143 & 1.331973873000 & 0.893823428997 & 0.893823624800 \\
\hline 0.3 & 1.354102768524 & 1.354102583000 & 1.000208677394 & 1.000208771000 \\
\hline 0.4 & 1.330095316143 & 1.330095126000 & 1.119123022586 & 1.119122920000 \\
\hline 0.5 & 1.259716623148 & 1.259716980000 & 1.238564988790 & 1.238564225000 \\
\hline 0.6 & 1.152840256063 & 1.152845461000 & 1.342772096989 & 1.342768205000 \\
\hline 0.7 & 1.026594271894 & 1.026627603000 & 1.416793455406 & 1.416793581000 \\
\hline 0.8 & 0.899120517439 & 0.899244376100 & 1.451520906161 & 1.451521131000 \\
\hline 0.9 & 0.784059292436 & 0.784362541500 & 1.445978046355 & 1.445913412000 \\
\hline 1.0 & 0.688601146327 & 0.688090398100 & 1.405950208844 & 1.405941241000 \\
\hline
\end{tabular}


Example 2 Suppose $\alpha$ is the per capita reduction in prey per predator and $\beta$ is the death rate per encounter of prey due to predation [24].

$$
\left\{\begin{array}{c}
\text { when }(\alpha>\beta) \\
\alpha=0.100 \\
\beta=0.0014 \\
\omega=0.080 \\
\tau=0.0012 \\
\lambda=-1.000 \\
x_{0}=4.000 \\
y_{0}=9.000
\end{array}\right.
$$$$
\left\{\begin{array}{c}
\text { when }(\alpha<\beta) \\
\alpha=0.0014 \\
\beta=0.100 \\
\omega=0.080 \\
\tau=0.0012 \\
\lambda=-1.000 \\
x_{0}=4.000 \\
y_{0}=9.000
\end{array}\right.
$$$$
\left\{\begin{array}{c}
\text { when }(\alpha=\beta) \\
\alpha=0.100 \\
\beta=0.100 \\
\omega=0.080 \\
\tau=0.0012 \\
\lambda=-1.000 \\
x_{0}=4.000 \\
y_{0}=9.000
\end{array}\right.
$$

Substitute the above parameters into algorithm (11), we have the following solutions:

Table 4: $(\alpha>\beta)$

\begin{tabular}{|c|c|c|c|c|}
\hline $\mathrm{t}$ & $\begin{array}{c}\text { Analytical } \\
\text { Solution } x(t)\end{array}$ & $\begin{array}{c}\text { VIM } \\
\text { Solution } x(t)\end{array}$ & $\begin{array}{c}\text { Analytical } \\
\text { Solution } y(t)\end{array}$ & $\begin{array}{c}\text { VIM } \\
\text { Solution } y(t)\end{array}$ \\
\hline 0.0 & 4.000000000000 & 4.000000000000 & 9.000000000000 & 9.000000000000 \\
\hline 0.1 & 4.035132287030 & 4.035132286000 & 8.932592638256 & 8.932592638000 \\
\hline 0.2 & 4.070611403006 & 4.070611402000 & 8.865727698739 & 8.865727700000 \\
\hline 0.3 & 4.106440756278 & 4.106440756000 & 8.799400925152 & 8.799400926000 \\
\hline 0.4 & 4.142623789899 & 4.142623790000 & 8.733608094714 & 8.733608095000 \\
\hline 0.5 & 4.179163981615 & 4.179163982000 & 8.668345018151 & 8.668345018000 \\
\hline 0.6 & 4.216064844061 & 4.216064844000 & 8.603607539534 & 8.603607540000 \\
\hline 0.7 & 4.253329953021 & 4.253329924000 & 8.539391509681 & 8.539391537000 \\
\hline 0.8 & 4.290962896869 & 4.290962804000 & 8.475692832270 & 8.475692921000 \\
\hline 0.9 & 4.328967276114 & 4.328967104000 & 8.412507466043 & 8.412507636000 \\
\hline 1.0 & 4.367346731478 & 4.367346480000 & 8.349831399216 & 8.349831655000 \\
\hline
\end{tabular}

Table 5: $(\alpha<\beta)$

\begin{tabular}{|c|c|c|c|c|}
\hline $\mathrm{t}$ & $\begin{array}{c}\text { Analytical } \\
\text { Solution } x(t)\end{array}$ & $\begin{array}{c}\text { VIM } \\
\text { Solution } x(t)\end{array}$ & $\begin{array}{c}\text { Analytical } \\
\text { Solution } y(t)\end{array}$ & $\begin{array}{c}\text { VIM } \\
\text { Solution } y(t)\end{array}$ \\
\hline 0.0 & 4.000000000000 & 4.000000000000 & 9.000000000000 & 9.000000000000 \\
\hline 0.1 & 3.657473020744 & 3.657473258000 & 8.932387296668 & 8.932387294000 \\
\hline 0.2 & 3.346536796070 & 3.346536998000 & 8.864935239948 & 8.864935237000 \\
\hline 0.3 & 3.064097573818 & 3.064097837000 & 8.797679574554 & 8.797679571000 \\
\hline 0.4 & 2.807379952412 & 2.807380177000 & 8.730651950326 & 8.730651947000 \\
\hline 0.5 & 2.573892073952 & 2.573892359000 & 8.663880372174 & 8.663880368000 \\
\hline 0.6 & 2.361396256269 & 2.361396506000 & 8.597389579842 & 8.597389576000 \\
\hline 0.7 & 2.167881361179 & 2.167881643000 & 8.531201405234 & 8.531201402000 \\
\hline 0.8 & 1.991539481074 & 1.991539751000 & 8.465335073997 & 8.465335070000 \\
\hline 0.9 & 1.830744135389 & 1.830744404000 & 8.399807487591 & 8.399807484000 \\
\hline 1.0 & 1.684031442829 & 1.684031744000 & 8.334633466917 & 8.334633463000 \\
\hline
\end{tabular}

Table 6: $(\alpha=\beta)$

\begin{tabular}{|c|c|c|c|c|}
\hline $\mathrm{t}$ & $\begin{array}{c}\text { Analytical } \\
\text { Solution } x(t)\end{array}$ & $\begin{array}{c}\text { VIM } \\
\text { Solution } x(t)\end{array}$ & $\begin{array}{c}\text { Analytical } \\
\text { Solution } y(t)\end{array}$ & $\begin{array}{c}\text { VIM } \\
\text { Solution } y(t)\end{array}$ \\
\hline 0.0 & 4.000000000000 & 4.000000000000 & 9.000000000000 & 9.000000000000 \\
\hline 0.1 & 3.693713901517 & 3.693714070000 & 8.932407277368 & 8.932407275000 \\
\hline
\end{tabular}


Falade, K. I et al./ NIPES Journal of Science and Technology Research 2(2) 2020 pp. $166-177$

\begin{tabular}{|l|l|l|l|l|}
\hline 0.2 & 3.413183652004 & 3.413183941000 & 8.865010279466 & 8.865010275000 \\
\hline 0.3 & 3.156082329242 & 3.156082424000 & 8.797838192760 & 8.797838190000 \\
\hline 0.4 & 2.920304355828 & 2.920304706000 & 8.730917063216 & 8.730917058000 \\
\hline 0.5 & 2.703946085963 & 2.703946207000 & 8.664270073277 & 8.664270071000 \\
\hline 0.6 & 2.505282320509 & 2.505282688000 & 8.597917875002 & 8.597917871000 \\
\hline 0.7 & 2.322752234866 & 2.322752401000 & 8.531878791541 & 8.531878789000 \\
\hline 0.8 & 2.154939736038 & 2.154940050000 & 8.466169095953 & 8.466169091000 \\
\hline 0.9 & 2.000562151892 & 2.000562388000 & 8.400803173218 & 8.400803170000 \\
\hline 1.0 & 1.858455074810 & 1.858455294000 & 8.335793735914 & 8.335793731000 \\
\hline
\end{tabular}

\section{Graphs Representation}

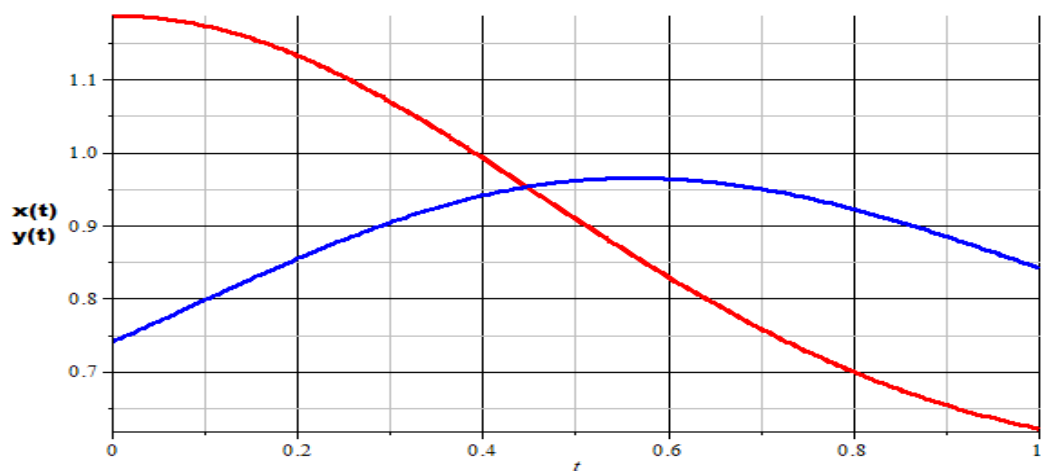

$-\mathbf{x}(\mathbf{t})-\mathbf{y}(\mathbf{t})$

Figure 1 Numerical solutions of the Predator $y(t)$ and Prey $x(t)$ when $\alpha<\beta$ Example 1

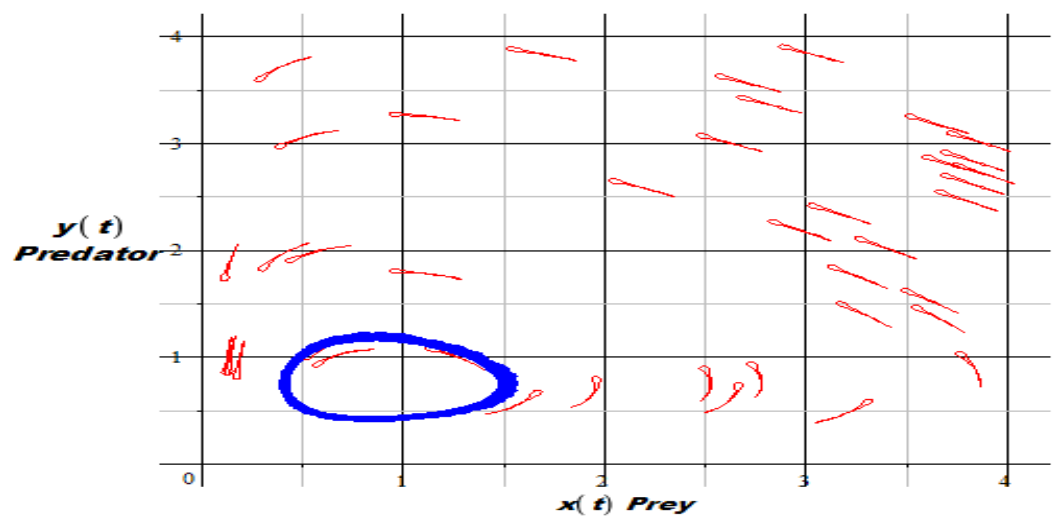

Figure 2 Unsteady states of phase planes for Predator-Prey when $\alpha<\beta$ Example 1

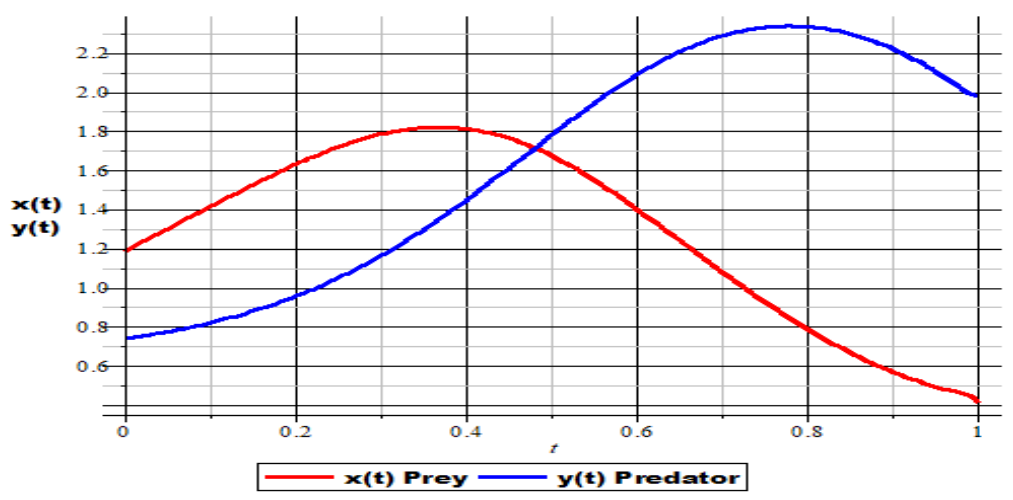

Figure 3 Numerical solutions of the Predator $y(t)$ and Prey $x(t)$ when $\alpha>\beta$ Example 1 


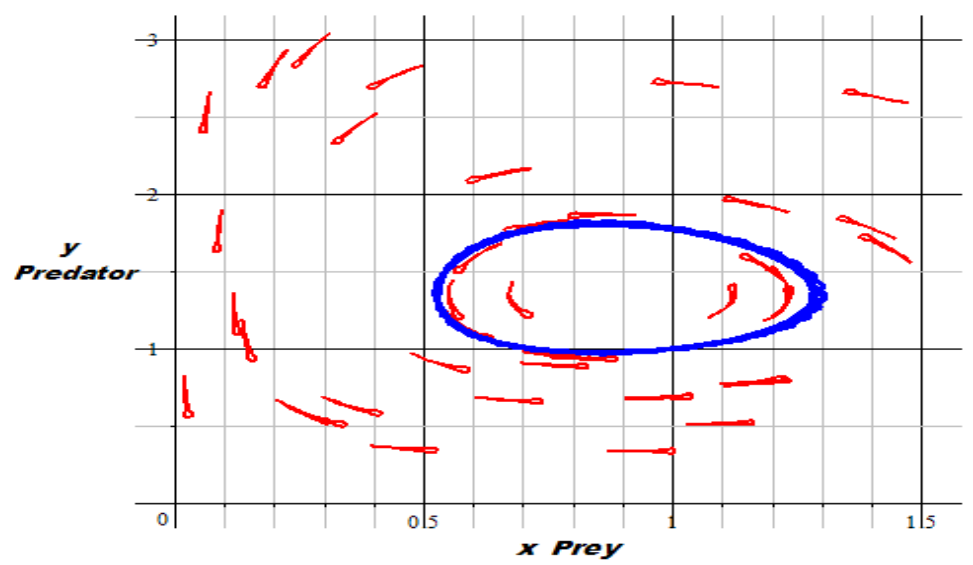

Figure 4 Steady states of phase planes for Predator-Prey when $\alpha>\beta$ Example 1

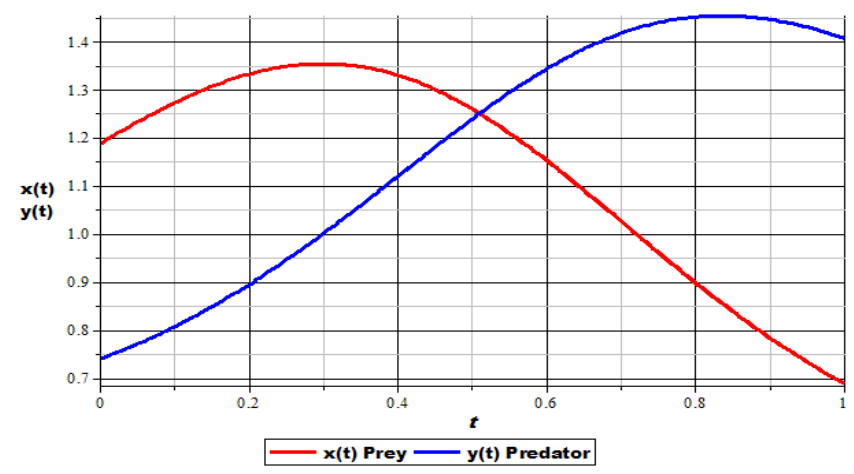

Figure 5 Numerical solutions of the Predator $y(t)$ and Prey $x(t)$ when $\alpha=\beta$ Example 1

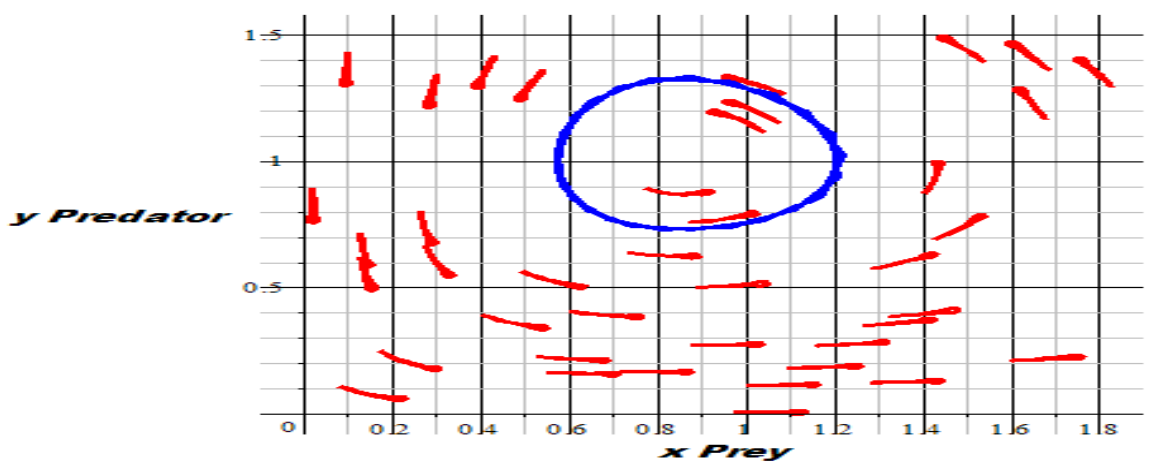

Figure 6 Steady states of phase planes for Predator-Prey when $\alpha=\beta$ Example 1

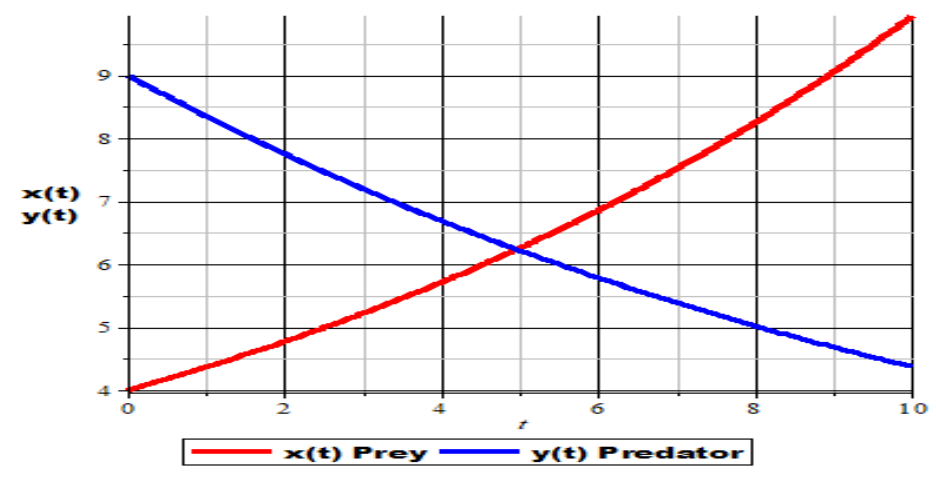

Figure 7 Numerical solutions of the Predator $y(t)$ and Prey $x(t)$ when $\alpha>\beta$ Example 2 
Falade, K. I et al./ NIPES Journal of Science and Technology Research 2(2) 2020 pp. $166-177$

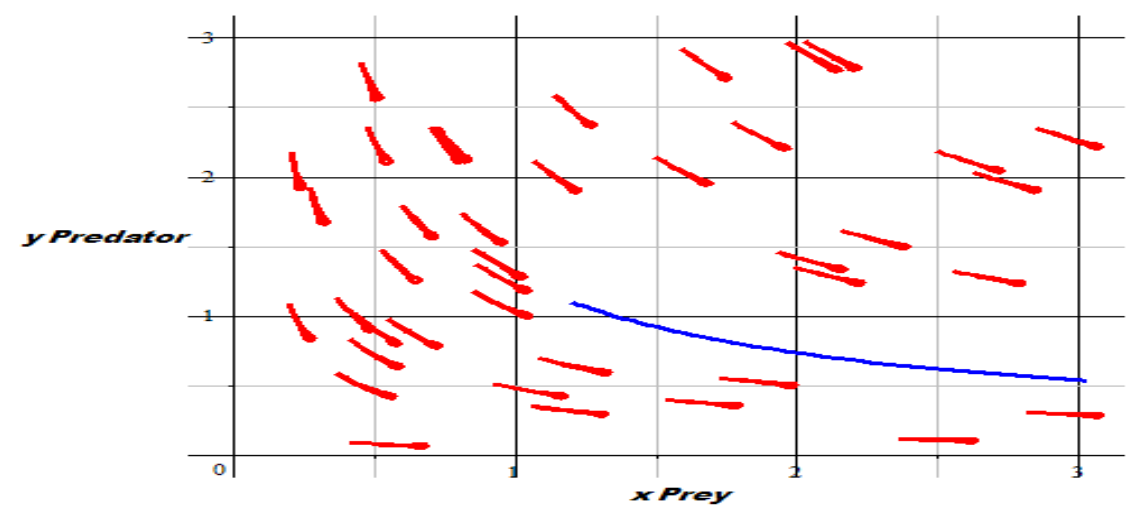

Figure 8 Unmutualistic interactions phase for Predator-Prey when $\alpha>\beta$ Example 2

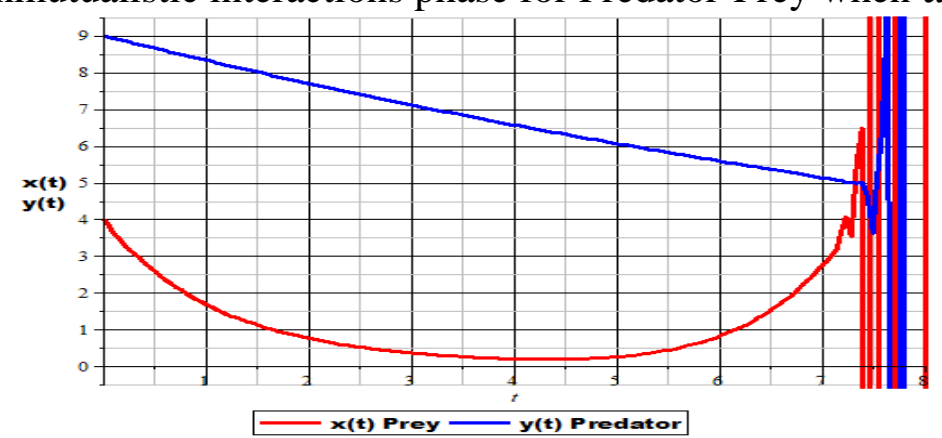

Figure 9 Numerical solutions of the Predator $y(t)$ and Prey $x(t)$ when $\alpha<\beta$ Example 2

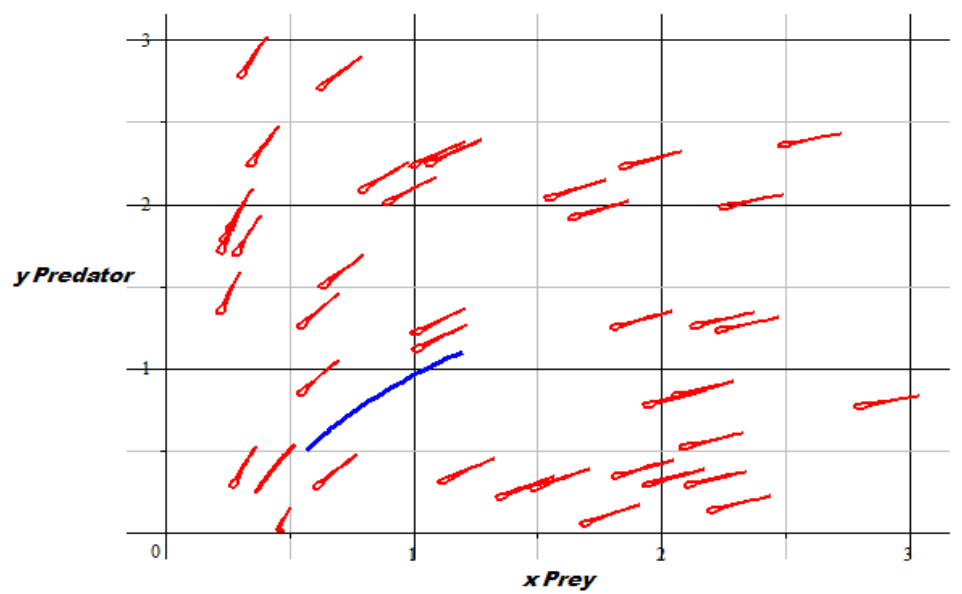

Figure 10 Mutualistic interactions phase for Predator-Prey when $\alpha<\beta$ Example 2

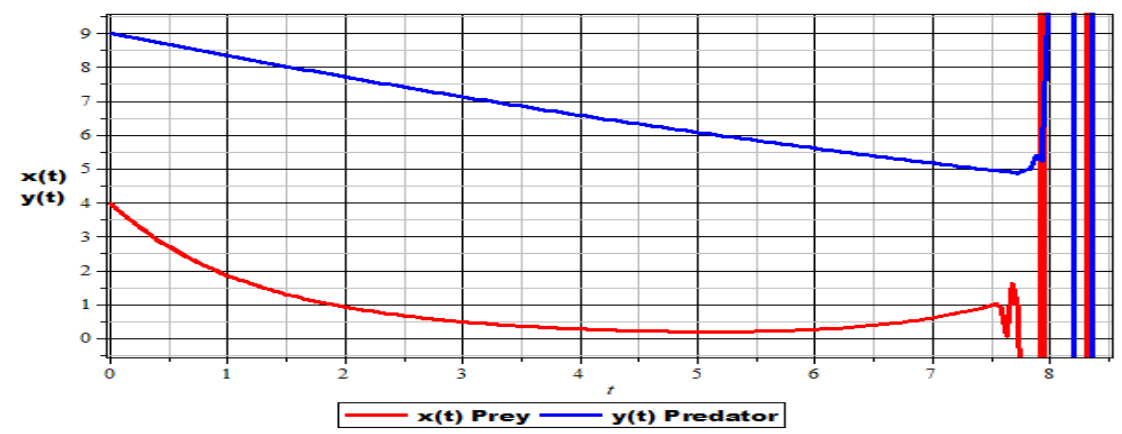


Figure 11 Numerical solutions of the Predator $y(t)$ and Prey $x(t)$ when $\alpha=\beta$ Example 2

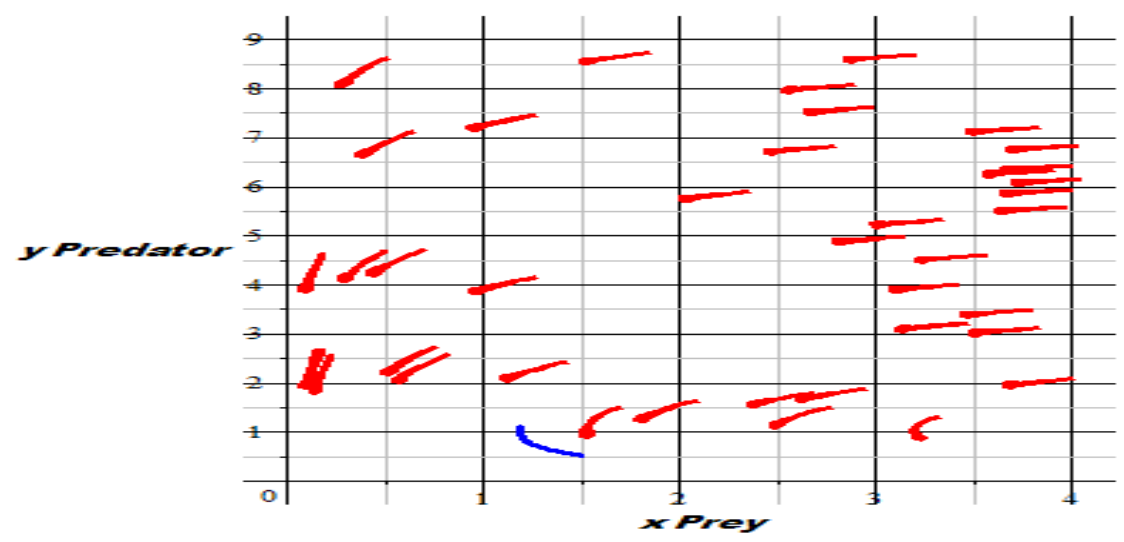

Figure 12 Unmutualistic interactions phase for Predator-Prey when $\alpha=\beta$ Example 2

\subsection{Discussion}

The numerical solutions for six cases of interaction behaviors between Predator-Prey are presented in Tables 1 to 6 while Figures 1 to 6 show plots relationship of Predator-Prey for example one and Figures 7 to 12 depict the numerical solution and interaction behaviors of Predator-Prey for example two. Finally, the study have revealed the interactions behaviors between two species (steady, unsteady, mutualistic and unmutualistic interactions) from computational analysis.

\section{Conclusion}

This article highlights the feasibility and capability of MAPLE 18 software codes to solve nonlinear system of Lotka-Volterra two species of Predator-Prey model. The conventional variation iteration method was employed using MAPLE 18 software commands to overcome the rigorous computational work and simplification of integrals involves during iteration process. Two examples are consider to test the efficiency of the algorithm and the numerical results obtained were compared with analytical solutions with little relative errors. The advantage of the MCVIM over the conventional approach is faster, easy and it provides an efficient numerical solutions. Finally, the proposed scheme is easy to implement and shows a good agreement with analytic results.

\section{References}

[1] H. F. Karwan. Jwamer and Aram M. Rashid (2012) New Technique For Solving System of First Order Linear Differential Equations Applied Mathematical Sciences, Vol. 6, no. 64, 3177 - 3183.

[2] V. Marinca and Nicolae H, (2012) Optimal Parametric Iteration Method for Solving Multispecies LotkaVolterra Equations Hindawi Publishing Corporation Discrete Dynamics in Nature and Society Volume 2012, Article ID 842121,pp 10-21.

[3] J.H He, (2000)A coupling method of a Homotopy technique and a perturbation technique for non-linear problems, International Journal of Non-Linear Mechanics, vol. 35, no. 1, pp. 37-43.

[4] F. Stephenie (2014) Predator-Prey Model Lecture note http://cs.unm.edu/ forrest

[5] M.S .Giovanni, R. Daniele (2003) A New Method for the Explicit Integration of Lotka-Volterra Equations Divulgaciones Matem’aticas Vol. 11 No. 1, pp. 1-17.

[6] F. Hosseini, M. Shekarab, M. Khodabin (2016) Numerical solutions of stochastic Lotka-Volterra equations via operational matrices Journal of Interpolation and Approximation in Scientific Computing SI.1 (2016) 37-42.

[7] C.G .Zhu, Yin. (2009).On competitive Lotka-Volterra model in random environments, Journal of Mathematical Analysis and Applications, 357(1), 154-170 
[8] H.Vahidin, Midhat. M,Jasmin Bektešević (2017) Lotka-Volterra Model with Two Predators and Their Prey TEM Journal. Volume 6, Issue 1, Pages 132-136.

[9] S Alebraheem,. Hussain, F. Ahmad, K. Nimer (2020) Application of differential transformation method for solving prey predator model with holling type 1 Italian Journal of Pure and Applied Mathematics ISSN22390227 pp 115-127.

[10] D. Venu, R. Gopala (2011)A Study on Series Solutions of Two Species Lotka Volterra Equations by Adomian Decomposition and Homotopy Perturbation Methods Gen. Math. Notes, Vol. 3, No. 2 pp. 13-26 ISSN 22197184; Copyright (C ICSRS Publication, 2011www.i-csrs.org

[11] A.Veronica, B. Mostafa and S.Mauricio (2010) Mathematical and numerical analysis for Predator-prey system in a polluted Networks and heterogeneous media doi:10.3934/nhm.2010.5.813 American institute of mathematical sciences volume 5 , number 4 .

[12]J.H He. (2000) Variational iteration method for autonomous ordinary differential systems. Applied Mathematics and Computation, 118 (2-3), 115-123.

[13]J.H He. (1999). A new approach to nonlinear partial differential equation. Communication in Nonlinear Science and Numerical Simulation, 2(4), 230-235.

[14] J.H He. (1998) A variational approach to nonlinear problems and its application. Mech. Applc., 20(1), 30-31.

[15] M. Inokuti, Sekine, H., T. Mura. (1978). General use of the Lagrange multiplier in nonlinear mathematical physics, In: S.Nemat-Nassed (ed.),Variational Method in the Mechanics of solids. Pergemon Press, 156-162

[16] J.H He (2006). Some asymptotic methods for strongly nonlinear equation. International Journal of Modern Physics A, B20 (10), 1141-1199.

[17] N. Bildik, A.Konuralp (2006) The use of variational iteration method, differential transform and Adomian decomposition method for solving different types of nonlinear partial differential equation. International Journal of Nonlinear Sciences and Numerical Simulation, 7(1), 65-70.

[18] Abdulwaf, E.M., Abdou, M.A., Mahmoud, A.A. (2006) The solution of nonlinear coagulation problem with mass loss. Chaos Solutions and Fractals, 26, 313-330.

[19]J.H He (1999) Variational iteration method a kind of nonlinear analytical technique: Some examples. International Journal of Non-Linear Mechanics, 34, 669-708.

[20] J.H He, J.H (2007) The variational iteration method for eight-order initial boundary value problems. Physical Scripta, 76, 680-682.

[21] Inokuti, M., Sekine, H., Mura, T.(1978) General use of the Lagrange multiplier in nonlinear mathematical physics, S.Nemat-Nassed (ed.),Variational Method in the Mechanics of solids. Pergemon Press, 156-162.

[22] Abbasbandy, S., Shivanian, E. (2009) Application of the variational iteration method for system of nonlinear Volterra's integro -differential equations. Mathematical and Computational Applications, 14 (2), 147-158.

[23] Stephanie Forrest (2000) Predator-Prey Models Dept. of Computer Science Univ. of New Mexico Albuquerque, NM http://cs.unm.edu forrestforrest@cs.unm.edu pp13-15.

[24] P. Susmita, P.M Sankar, B. Paritosh B. (2016) Numerical solution of Lotka Volterra prey predator model by using Runge-Kutta-Fehlberg method and Laplace Adomian decomposition method, Alexandria Eng. J. University http://dx.doi.org/10.1016/j.aej.2015.12.026. 